\title{
Advances in S/TEM Sample Preparation Using a FIB-SEM: Techniques for the Ultimate Sample
}

\author{
B. Van Leer, D. Wall \\ FEI Company, 5350 NE Dawson Creek Drive, Hillsboro, Oregon, 97124 USA
}

Over the past 19 years, focused ion beam (FIB) and SEM/FIB instrumentation has transformed scientists' ability to investigate materials to develop new sample preparation methods to becoming the industry standard and "work-horse" for site-specific cross-section analysis, TEM/STEM sample preparation (cross-section or plan view) and nanoscale patterning/prototyping applications. Early FIBs allowed the researcher to generate TEM samples in conjunction with the tripod polishing method in a relatively short time frame (approximately 2 to 3 hours) [1]. Now, state-of-the-art FIBs and SEM/FIBs provide many times more current density than 15 years ago and offer in-situ lift-out (INLO) techniques that allow the user to prepare a sample to TEM/STEM transparency in as little as 20-30 minutes [2]. With the addition of integrated digital pattern generators, FIB and SEM/FIB, engineers can develop direct-write nanoprototyping processes from sub-micron to nanoscale without the use of external pattern generators [3]. Systems with this functionality generally have improved approaches for sample preparation because of novel milling strategies that can be built into the user interface and a finer and more dynamic level of control over the patterning system. Automated sample preparation extends the usability of the system allowing for overnight sample preparation runs, which can achieve TEM sample thicknesses of $80 \mathrm{~nm}$ with $>80 \%$ reliability and allows the usability of the system far beyond the 8-hour workday. In this paper, we present advances in TEM sample preparation using SEM/FIB instruments and discuss how these advances enable high quality samples.

With demand for TEM sample preparation at an all time high, electron microscopists and researchers require sample generation to take much less than a day to prepare. There are many factors that enable a system's ease-of-use and flexibility. These factors include optimal beam parameters, system stability and software functionality including automation and novel approaches to sample preparation. Recent advances in all four have enabled SEM/FIB operators to routinely generate high quality, site specific samples in 2 hours or less. For non-site specific materials the time can be reduced to much less than an hour. Balancing spot size and beam current density allows thin, uniform lamella formation to occur in minutes. The first step in TEM specimen preparation is making an SEM-type cross-section. One way to speed up specimen preparation is to use higher beam currents that still maintain good current density profiles. As TEM sample preparation with FIBs has become more mainstream, the quality of sample preparation has also become increasingly important. Sample damage with high energy FIB is well characterized among many materials. Recently, the use of low energy FIB to reduce the damage continues to generate interest in the scientific community [4]. FIBs can now routinely operate in a low energy regime with imaging capabilities that allow for site specific pattern placement onto samples. Figure 1 reveals an example of $2 \mathrm{keV}$ and $1 \mathrm{keV} \mathrm{Ga}{ }^{+}$focused ion beam imaging performance with resulting material damage. In addition to importance of eliminating damage caused by the preparation technique, hitting a site-specific region of the target is also crucial. To that end, there have been many new end-pointing techniques, which allow the operator to watch the bulk milling and lamella formation process to maintain control of 
the milling process and provide visibility of the target structure. These techniques include simultaneous imaging while patterning, sequential image and mill, and FIB real time monitoring. Each of these methods has strengths during the preparation process and each will be discussed. Lastly, corrected S/TEM microscopes require ultra thin $(<45 \mathrm{~nm}$ thick) specimens to fully take advantage of the microscopes capabilities. The approach to finishing a thin specimen has evolved over the past 5 years to include novel approaches such as inverting the sample prior to final polish.

\section{References}

[1] S.J. Klepeis et al., “A Grinding/Polishing Tool for SEM Sample Preparation.” In Specimen Preparation for Transmission Electron Microscopy of Materials, Mater. Res. Soc. Proc. 115 1987.

[2] D. Wall, "Ultra-Fast In-Situ S/TEM Sample Preparation.” FEI Company Application Note P/N 04AP-FR0111, FEI Company, 2007.

[3] B. Van Leer et al., "Applications of Focused Ion Beam and DualBeam for Nanofabrication." Scanning Microscopy for Nanotechnology: Techniques and Applications, Springer, New York, 2007

[4] L. A. Giannuzzi et al., Micros. Microanal., 11(Suppl 2) (2005) 828.
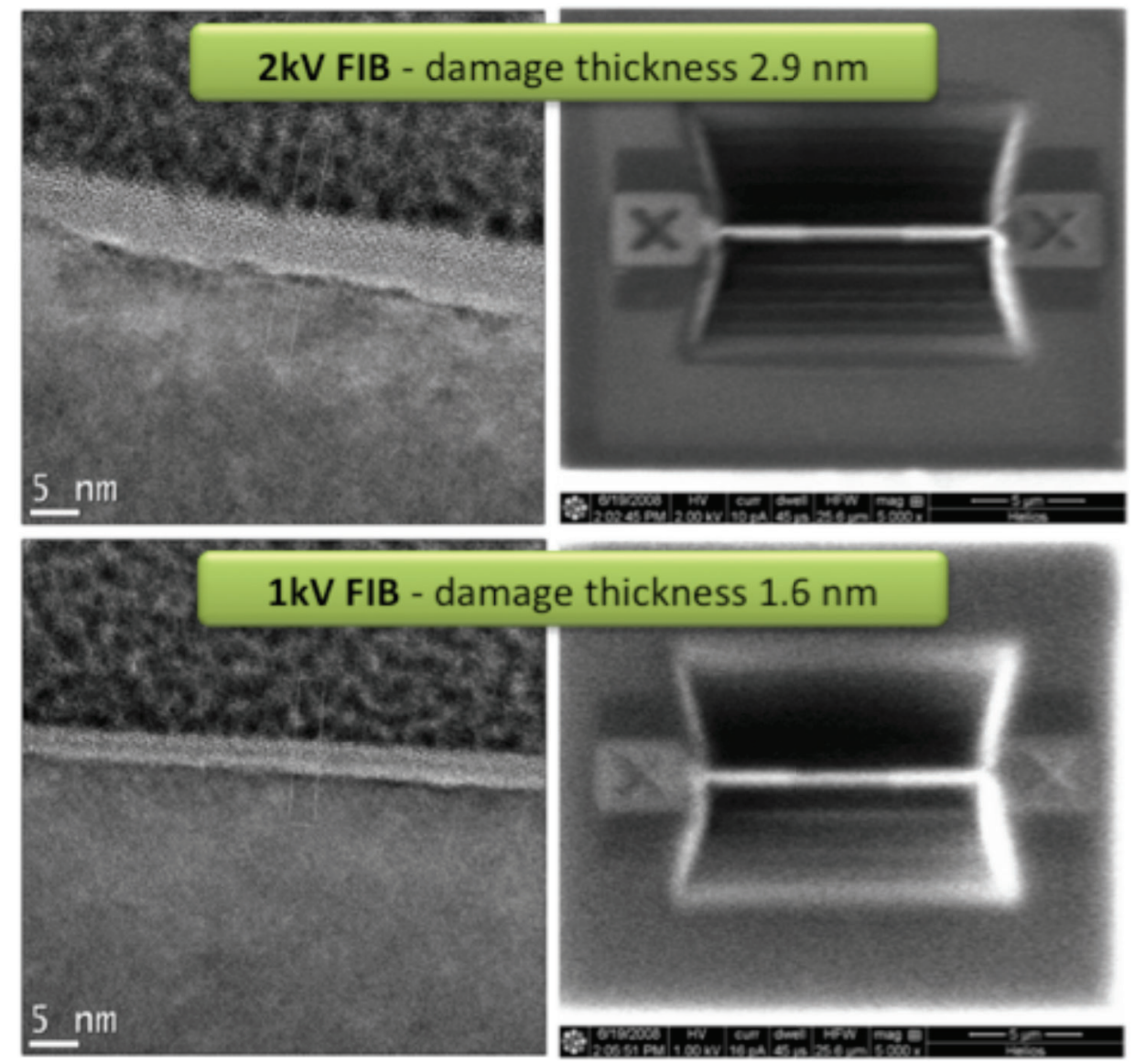

Figure 1. FIB damage in Silicon and FIB induced secondary-electron imaging performance for 2 $\mathrm{keV}$ and $1 \mathrm{keV} \mathrm{Ga}{ }^{+}$FIB beam 